\title{
Integration by parts and its applications of a new nonlocal fractional derivative with Mittag-Leffler nonsingular kernel
}

\author{
Thabet Abdeljawada ${ }^{\mathrm{a}}$, Dumitru Baleanu ${ }^{\mathrm{b}, \mathrm{c}, *}$ \\ ${ }^{a}$ Department of Mathematics and Physical Sciences, Prince Sultan University, P. O. Box 66833, 11586 Riyadh, Saudi Arabia. \\ ${ }^{b}$ Department of Mathematics, Çankaya University, 06530 Ankara, Turkey. \\ ${ }^{c}$ Institute of Space Sciences, Magurele-Bucharest, Romania.
}

Communicated by Sh. Wu

\begin{abstract}
In this manuscript we define the right fractional derivative and its corresponding right fractional integral for the newly suggested nonlocal fractional derivative with Mittag-Leffler kernel. Then, we obtain the related integration by parts formula. We use the Q-operator to confirm our results. The related Euler-Lagrange equations are reported and one illustrative example is discussed. (C)2017 All rights reserved.
\end{abstract}

Keywords: Fractional calculus, Mittag-Leffler function, fractional integration by parts, fractional Euler-Lagrange equations. 2010 MSC: 47H10, 54H25.

\section{Introduction and preliminaries}

Fractional calculus is developing faster during the last few years and many phenomena possessing the power law effect were described accurately with fractional models (see for example Refs. [7, 14, 17, 18, 20, $21,23,24,26,27]$ and the references therein). Many excellent results of the fractional models were reported in various fields of science and engineering. One of the specificity of the fractional calculus is that we have many fractional derivatives which gives the researcher the opportunity to choose the specific fractional derivative which corresponds better to a given real world problem. The description of phenomena with memory effect is still a big challenge for the researchers, therefore new tools and methods should be created to be able to get better description of the real world phenomena and the existing models. In this respect it seems that there is a need of new fractional derivatives with nonsingular kernel. One of the best candidates among the existing kernels is the one based on Mittag-Leffler (ML) functions [9]. Based on this, very recently a new fractional derivative [9] was constructed and applied to several real world problems $[8,10]$. Some results on the discrete version of this new fractional derivative can be seen in [6]. For the first nonlocal fractional derivatives with nonsingular exponential kernel we refer to $[12,13,19,25]$ and the references therein and for other local approaches of the fractional derivatives we refer to the

\footnotetext{
*Corresponding author

Email addresses: tabdeljawad@psu.edu.sa (Thabet Abdeljawad), dumitru@cankaya.edu.tr (Dumitru Baleanu)
} 
recent manuscripts $[3,15]$. In this paper we would like to present several important properties of the new derivative introduced in [9] in order to see the advantages of it as well as in order to start to apply it in fractional variational principles and optimal control problems. Having above mentioned things in mind we present in the second section the fundamental integration by parts formula. Integration by parts is of great importance in fractional calculus [17] and discrete fractional calculus $[1,2,4,5]$. In the third section we developed the corresponding fractional Euler-Lagrange equations [11] and we give an illustrative example of it.

From the classical fractional calculus, we recall $[17,23,24]$

- The left Riemann-Liouville fractional integral of order $\alpha>0$ starting from a has the following form

$$
\left({ }_{a} I^{\alpha} f\right)(t)=\frac{1}{\Gamma(\alpha)} \int_{a}^{t}(t-s)^{\alpha-1} f(s) d s .
$$

- The right Riemann-Liouville fractional integral of order $\alpha>0$ ending at $b>a$ is defined by

$$
\left(I_{b}^{\alpha} f\right)(t)=\frac{1}{\Gamma(\alpha)} \int_{t}^{b}(s-t)^{\alpha-1} f(s) d s .
$$

- The left Riemann-Liouville fractional derivative of order $0<\alpha<1$ starting at a has the form

$$
\left({ }_{a} D^{\alpha} f\right)(t)=\frac{d}{d t}\left({ }_{a} I^{1-\alpha} f\right)(t) .
$$

- The right Riemann-Liouville fractional derivative of order $0<\alpha<1$ ending at $b$ becomes

$$
\left(D_{b}^{\alpha} f\right)(t)=\frac{-d}{d t}\left(I_{b}^{1-\alpha} f\right)(t)
$$

\section{The right fractional derivative and integration by parts formula}

If $f$ is defined on an interval $[a, b]$, then the action of the Q-operator is defined as $(Q f)(t)=f(a+b-t)$. Definition 2.1 ([9]). Let $f \in H^{1}(a, b), a<b$, and $\alpha \in[0,1]$, then the definition of the new (left Caputo) fractional derivative with Mittag-Leffler nonsingular kernel becomes:

$$
\left({ }_{a}^{A B C} D^{\alpha} f\right)(t)=\frac{B(\alpha)}{1-\alpha} \int_{a}^{t} f^{\prime}(x) E_{\alpha}\left[-\alpha \frac{(t-x)^{\alpha}}{1-\alpha}\right] d x
$$

and in the left Riemann-Liouville sense has the following form:

$$
\left(\begin{array}{l}
A B R \\
a
\end{array} D^{\alpha} f\right)(t)=\frac{B(\alpha)}{1-\alpha} \frac{d}{d t} \int_{a}^{t} f(x) E_{\alpha}\left[-\alpha \frac{(t-x)^{\alpha}}{1-\alpha}\right] d x,
$$

where $\mathrm{B}(0)=\mathrm{B}(1)=1$. The associated fractional integral is

$$
\left({ }_{a}^{A B} I^{\alpha} f\right)(t)=\frac{1-\alpha}{B(\alpha)} f(t)+\frac{\alpha}{B(\alpha)}\left({ }_{a} I^{\alpha} f\right)(t) .
$$

Let us denote the new right Riemann-Liouville fractional derivative that we wish to propose by ${ }^{A B R} D_{b}^{\alpha}$ and its corresponding integral by ${ }^{A B} I_{b}^{\alpha}$. From classical fractional calculus it is known that $\left({ }_{a} I^{\alpha} Q f\right)(t)=$ $Q\left(I_{b}^{\alpha} f\right)(t)$ and $\left({ }_{a} D^{\alpha} Q f\right)(t)=Q\left(D_{b}^{\alpha} f\right)(t)$. We wish this relation to be satisfied for the new left and right fractional derivatives and integrals.

$$
\begin{aligned}
\left({ }_{a}^{A B R} D^{\alpha} Q f\right)(t) & =\frac{B(\alpha)}{1-\alpha} \frac{d}{d t} \int_{a}^{t} f(a+b-x) E_{\alpha}\left[-\alpha \frac{(t-x)^{\alpha}}{1-\alpha}\right] d x \\
& =\frac{B(\alpha)}{1-\alpha} \frac{d}{d t} \int_{a+b-t}^{b} f(u) E_{\alpha}\left[-\alpha \frac{(u-(a+b-t))^{\alpha}}{1-\alpha}\right] d x,
\end{aligned}
$$

where the change of variable $u=a+b-x$ is used. Based on the relation (2.2) we formulate the definition 
for the new right fractional derivative.

Definition 2.2. The right fractional new derivative with ML kernel of order $\alpha \in[0,1]$ is defined by

$$
\left({ }^{A B R} D_{b}^{\alpha} f\right)(t)=-\frac{B(\alpha)}{1-\alpha} \frac{d}{d t} \int_{t}^{b} f(x) E_{\alpha}\left[-\alpha \frac{(x-t)^{\alpha}}{1-\alpha}\right] d x .
$$

On the other hand,

$$
\begin{aligned}
\left({ }_{a}^{A B} I^{\alpha} Q f\right)(t) & =\frac{1-\alpha}{B(\alpha)} f(a+b-t)+\frac{\alpha}{B(\alpha)}\left({ }_{a} I^{\alpha} Q f\right)(t) \\
& =\frac{1-\alpha}{B(\alpha)} f(a+b-t)+\frac{\alpha}{B(\alpha)} Q\left(I_{b}^{\alpha} f\right)(t)=Q\left[\frac{1-\alpha}{B(\alpha)} f(t)+\frac{\alpha}{B(\alpha)}\left(I_{b}^{\alpha} f\right)(t)\right] .
\end{aligned}
$$

Moreover, we solve the equation $\left({ }^{A B} D_{b}^{\alpha} f\right)(t)=u(t)$. Indeed,

$$
\left({ }^{A B} D_{b}^{\alpha} f\right)(t)=\left({ }^{A B} D_{b}^{\alpha} Q Q f\right)(t)=\left(Q^{A B}{ }_{a} D^{\alpha} Q f\right)(t)=u(t),
$$

or

$$
\left({ }_{a}^{A B} D^{\alpha} Q f\right)(t)=Q u(t)
$$

and hence,

$$
\mathrm{Q} f(t)=\frac{1-\alpha}{B(\alpha)} \mathrm{Qu}(\mathrm{t})+\frac{\alpha}{\mathrm{B}(\alpha)}{ }_{\mathrm{a}} \mathrm{I}^{\alpha} \mathrm{Qu}(\mathrm{t})=\frac{1-\alpha}{\mathrm{B}(\alpha)} \mathrm{Qu}(\mathrm{t})+\frac{\alpha}{\mathrm{B}(\alpha)} \mathrm{Q} \mathrm{I}_{\mathrm{b}}^{\alpha} \mathrm{u}(\mathrm{t})
$$

Applying $\mathrm{Q}$ to both sides above, we have

$$
f(t)=\frac{1-\alpha}{B(\alpha)} u(t)+\frac{\alpha}{B(\alpha)} I_{b}^{\alpha} u(t)
$$

Now, relations (2.3) and (2.4) suggest the following definition for the new right fractional integral.

Definition 2.3. The right fractional new integral with ML kernel of order $\alpha \in[0,1]$ is defined by

$$
\left({ }^{A B} I_{b}^{\alpha} f\right)(t)=\frac{1-\alpha}{B(\alpha)} f(t)+\frac{\alpha}{B(\alpha)} I_{b}^{\alpha} f(t)
$$

Before we present an integration by part formula for the new proposed fractional derivatives and integrals, we introduce the following function spaces: for $p \geqslant 1$ and $\alpha>0$, we define

$$
\left({ }_{a}^{A B} I^{\alpha}\left(L_{p}\right)=\left\{f: f={ }_{a}^{A B} I^{\alpha} \varphi, \varphi \in L_{p}(a, b)\right\}\right.
$$

and

$$
\left({ }^{A B} I_{b}^{\alpha}\left(L_{p}\right)=\left\{f: f={ }^{A B} I_{b}^{\alpha} \phi, \phi \in L_{p}(a, b)\right\} .\right.
$$

In [9] it was shown that the left fractional operator ${ }_{a}^{A B R} D^{\alpha}$ and its associate fractional integral ${ }_{a}^{A B} I^{\alpha}$ satisfy $\left({ }_{a}^{A B R} D^{\alpha}{ }_{a}^{A B} I^{\alpha} f\right)(t)=f(t)$ and above we have shown that $\left.\left({ }^{A B R} D_{b}^{\alpha} A B\right) I_{b}^{\alpha} f\right)(t)=f(t)$. On the other hand we next prove that $\left({ }_{a}^{A B} I^{\alpha} \underset{a}{A B R} D^{\alpha} f\right)(t)=f(t)$ and $\left({ }^{A B} I_{b}^{\alpha} A B R D_{b}^{\alpha} f\right)(t)=f(t)$ and hence the function spaces $\left({ }_{a}^{A B} I^{\alpha}\left(L_{p}\right)\right.$ and $\left({ }^{A B} I_{b}^{\alpha}\left(L_{p}\right)\right.$ are nonempty.

Theorem 2.4. The functions $\left({ }_{a}^{A B R} D^{\alpha} f\right)(t)$ and $\left({ }^{A B R} D_{b}^{\alpha} f\right)(t)$ satisfy the equations

$$
\left(\begin{array}{l}
A B \\
a
\end{array} I^{\alpha} g\right)(t)=f(t), \quad\left({ }^{A B} I_{b}^{\alpha} g\right)(t)=f(t),
$$

respectively.

Proof. We just prove the left case. The right case can be proved by means of the Q-operator. From the definition, the first equation is equivalent to 


$$
\frac{1-\alpha}{B(\alpha)} g(t)+\frac{\alpha}{B(\alpha)}\left({ }_{a} I^{\alpha} g\right)(t)=f(t)
$$

Apply the Laplace transform to see that

$$
\frac{1-\alpha}{B(\alpha)} G(s)+\frac{\alpha}{B(\alpha)} s^{-\alpha} G(s)=F(s)
$$

From which it follows that

$$
G(s)=\frac{B(\alpha)}{1-\alpha} \frac{F(s) s^{\alpha}}{s^{\alpha}+\frac{\alpha}{1-\alpha}} .
$$

Finally, the Laplace inverse will lead to that $g(t)=\left(a_{a}^{A B R} D^{\alpha} f\right)(t)$.

Theorem 2.5 (Integration by parts). Let $\alpha>0, p \geqslant 1, q \geqslant 1$, and $\frac{1}{p}+\frac{1}{q} \leqslant 1+\alpha(p \neq 1$ and $q \neq 1$ in the case $\left.\frac{1}{\mathrm{p}}+\frac{1}{\mathrm{q}}=1+\alpha\right)$. Then

- If $\varphi(x) \in \mathrm{L}_{p}(\mathrm{a}, \mathrm{b})$ and $\psi(\mathrm{x}) \in \mathrm{L}_{\mathrm{q}}(\mathrm{a}, \mathrm{b})$, then

$$
\begin{aligned}
\int_{a}^{b} \varphi(x)\left(\left(_{a}^{A B} I^{\alpha} \psi\right)(x) d x\right. & =\frac{1-\alpha}{B(\alpha)} \int_{a}^{b} \psi(x) \varphi(x) d x+\frac{\alpha}{B(\alpha)} \int_{a}^{b}\left(I_{b}^{\alpha} \varphi\right)(x) \psi(x) d x \\
& =\int_{a}^{b} \psi(x)\left({ }^{A B} I_{b}^{\alpha} \varphi\right)(x) d x
\end{aligned}
$$

and similarly,

$$
\int_{a}^{b} \varphi(x)\left({ }^{A B} I_{b}^{\alpha} \psi\right)(x) d x=\frac{1-\alpha}{B(\alpha)} \int_{a}^{b} \psi(x) \varphi(x) d x+\frac{\alpha}{B(\alpha)} \int_{a}^{b}\left({ }_{a} I^{\alpha} \varphi\right)(x) \psi(x) d x=\int_{a}^{b} \psi(x)\left({ }_{a}^{A B} I^{\alpha} \varphi\right)(x) d x .
$$

- If $\mathrm{f}(\mathrm{x}) \in^{\mathrm{AB}} \mathrm{I}_{\mathrm{b}}^{\alpha}\left(\mathrm{L}_{p}\right)$ and $\mathrm{g}(\mathrm{x}) \in{ }_{\mathrm{a}}^{\mathrm{AB}} \mathrm{I}^{\alpha}\left(\mathrm{L}_{q}\right)$, then

$$
\int_{a}^{b} f(x)\left(\left(_{a}^{A B R} D^{\alpha} g\right)(x) d x=\int_{a}^{b}\left({ }^{A B R} D_{b}^{\alpha} f\right)(x) g(x) d x .\right.
$$

Proof.

- From the definition and the integration by parts for (classical) Riemann-Liouville fractional integrals we have

$$
\begin{aligned}
\int_{a}^{b} \varphi(x)\left({ }_{a}^{A B} I^{\alpha} \psi\right)(x) d x & =\int_{a}^{b} \varphi(x)\left[\frac{1-\alpha}{B(\alpha)} \psi(x)+\frac{\alpha}{B(\alpha)}{ }_{a} I^{\alpha} \psi(x)\right] d x \\
& =\frac{1-\alpha}{B(\alpha)} \int_{a}^{b} \varphi(x) \psi(x) d x+\frac{\alpha}{B(\alpha)} \int_{a}^{b} \psi(x) I_{b}^{\alpha} \varphi(x) d x \\
& =\int_{a}^{b} \psi(x)\left[\frac{1-\alpha}{B(\alpha)} \varphi(x)+\frac{\alpha}{B(\alpha)} I_{b}^{\alpha} \varphi(x)\right] d x=\int_{a}^{b} \psi(x)\left({ }^{A B} I_{b}^{\alpha} \varphi(x)\right) d x .
\end{aligned}
$$

The other case follows similarly by Definition 2.3 and the integration by parts for (classical) RiemannLiouville fractional integrals.

- From definition and the first part we have

$$
\int_{a}^{b} f(x)\left({ }_{a}^{A B R} D^{\alpha} g\right)(x) d x=\int_{a}^{b}\left({ }^{A B} I_{b}^{\alpha} \phi\right)(x) \cdot\left({ }_{a}^{A B R} D^{\alpha} \circ{ }_{a}^{A B R} I^{\alpha} \varphi\right)(x) d x
$$




$$
\begin{aligned}
= & \int_{a}^{b}\left({ }^{A B} I_{b}^{\alpha} \phi\right)(x) \cdot \varphi(x) d x \\
= & \frac{1-\alpha}{B(\alpha)} \int_{a}^{b} \phi(x) \varphi(x) d x+\frac{\alpha}{B(\alpha)} \int_{a}^{b} \phi(x)\left({ }_{a} I^{\alpha} \varphi\right)(x) d x \\
= & \frac{1-\alpha}{B(\alpha)} \int_{a}^{b}\left({ }^{A B R} D_{b}^{\alpha} f\right)(x)\left({ }_{a}^{A B R} D^{\alpha} g\right) d x \\
& +\frac{\alpha}{B(\alpha)} \int_{a}^{b}\left({ }^{A B R} D_{b}^{\alpha} f\right)(x)\left[\frac{B(\alpha)}{\alpha} g(x)-\frac{1-\alpha}{\alpha}\left({ }_{a}^{A B R} D^{\alpha} g\right)\right] d x \\
= & \int_{a}^{b}\left({ }^{A B R} D_{b}^{\alpha} f\right)(x) g(x) d x .
\end{aligned}
$$

In the proof, the identity $\left({ }_{a} I^{\alpha} \varphi\right)(x)=\frac{B(\alpha)}{\alpha}\left({ }_{a}^{A B} I^{\alpha} \varphi\right)(x)-\frac{1-\alpha}{\alpha} \varphi(x)$ deriven from (2.1) has used.

Example 2.6. This example is a numerical application of Theorem 2.5.

- To verify (2.5), let $\psi(x)=x, \varphi(x)=1-x, \alpha=\frac{1}{2}$, [a, b] $=[0,1]$, and $B(\alpha)=1$. Then,

$$
{ }_{0}^{A B} I^{1 / 2} x=\frac{x}{2}+\frac{1}{2} \frac{\Gamma(2) x^{3 / 2}}{\Gamma(5 / 2)}=\frac{x}{2}+\frac{2 x^{3 / 2}}{3 \sqrt{\pi}}
$$

and

$$
{ }^{\mathrm{AB}} \mathrm{I}_{1}^{1 / 2}(1-x)=\frac{1-x}{2}+\frac{2(1-x)^{3 / 2}}{3 \sqrt{\pi}}
$$

Hence, the left hand side of (2.5) results in

$$
\int_{a}^{b} \varphi(x)\left({ }_{a B}^{A B} I^{\alpha} \psi\right)(x) d x=\int_{0}^{1}(1-x){ }^{A B}{ }_{0} I^{1 / 2} x=\int_{0}^{1}(1-x)\left[\frac{x}{2}+\frac{2 x^{3 / 2}}{3 \sqrt{\pi}}\right] d x=\frac{1}{12}+\frac{8}{105 \sqrt{\pi}}
$$

and

$$
\int_{a}^{b} \psi(x)\left({ }^{A B} I_{b}^{\alpha} \varphi(x) d x=\int_{0}^{1} x\left({ }^{A B} I_{1}^{1 / 2}(1-x) d x=\int_{0}^{1} x\left[\frac{1-x}{2}+\frac{2(1-x)^{3 / 2}}{3 \sqrt{\pi}}\right] d x=\frac{1}{12}+\frac{8}{105 \sqrt{\pi}} .\right.\right.
$$

- To verify the second part of Theorem 2.5 , let $f(x)=\frac{1-x}{2}+\frac{2(1-x)^{3 / 2}}{3 \sqrt{\pi}}$ and $g(x)=\frac{x}{2}+\frac{2 x^{3 / 2}}{3 \sqrt{\pi}}$ with $\alpha=\frac{1}{2}$, $[a, b]=[0,1]$, and $B(\alpha)=1$. Then,

$$
\int_{a}^{b} f(x)\left(a_{a}^{A B R} D^{\alpha} g\right)(x) d x=\int_{0}^{1}\left[\frac{1-x}{2}+\frac{2(1-x)^{3 / 2}}{3 \sqrt{\pi}}\right] x d x=\frac{1}{12}+\frac{8}{105 \sqrt{\pi}}
$$

and

$$
\int_{a}^{b}\left({ }^{A B R} D_{b}^{\alpha} f\right)(x) g(x) d x=\int_{0}^{1}(1-x)\left[\frac{x}{2}+\frac{2 x^{3 / 2}}{3 \sqrt{\pi}}\right] d x=\frac{1}{12}+\frac{8}{105 \sqrt{\pi}} .
$$

From [9] we recall the relation between the Riemann-Liouville and Caputo new derivatives as

$$
\left({ }_{0}^{A B C} D^{\alpha} f\right)(t)=\left({ }_{0}^{A B R} D^{\alpha} f\right)(t)-\frac{B(\alpha)}{1-\alpha} f(0) E_{\alpha}\left(-\frac{\alpha}{1-\alpha} t^{\alpha}\right) .
$$

From [16] recall the (left) generalized fractional integral operator

$$
\left(\mathbf{E}_{\rho, \mu, \omega, a^{+}}^{\gamma} \varphi\right)(x)=\int_{a}^{x}(x-t)^{\mu-1} E_{\rho, \mu}^{\gamma}\left[\omega(x-t)^{\rho}\right] \varphi(t) d t, \quad x>a .
$$


Analogously, the (right) generalized fractional integral operator can be defined by

$$
\left(\mathbf{E}_{\rho, \mu, \omega, b^{-}}^{\gamma} \varphi\right)(x)=\int_{x}^{b}(t-x)^{\mu-1} E_{\rho, \mu}^{\gamma}\left[\omega(t-x)^{\rho}\right] \varphi(t) d t, \quad x<b,
$$

where $E_{\rho, \mu}^{\gamma}(z)=\sum_{k=0}^{\infty} \frac{(\gamma)_{k} z^{k}}{\Gamma(\rho k+\mu) k !}$, denotes the generalized Mittag-Leffler function defined for complex $\rho, \mu, \gamma(\operatorname{Re}(\rho)>0)[16,17]$.

Definition 2.7. The new (right) Caputo fractional derivative of order $0<\alpha<1$ is defined by

$$
\left({ }^{A B C} D_{b}^{\alpha} f\right)(t)=-\frac{B(\alpha)}{1-\alpha} \int_{t}^{b} f^{\prime}(x) E_{\alpha}\left[-\alpha \frac{(x-t)^{\alpha}}{1-\alpha}\right] d x .
$$

Next, we prove the right version of (2.6) by making use of the Q-operator.

Proposition 2.8. The right new Riemann-Liouville fractional derivative and the new right Caputo fractional derivative are related by the identity:

$$
\left({ }^{A B C} D_{b}^{\alpha} f\right)(t)=\left({ }^{A B R} D_{b}^{\alpha} f\right)(t)-\frac{B(\alpha)}{1-\alpha} f(b) E_{\alpha}\left(-\frac{\alpha}{1-\alpha}(b-t)^{\alpha}\right) .
$$

Proof. Applying the Q-operator to the identity (2.6) and using the dual facts

$$
\mathrm{Q}\left({ }_{0}^{A B R} D^{\alpha} f\right)(t)=\left({ }^{A B R} D_{b}^{\alpha} Q f\right)(t)
$$

and $Q\left({ }_{0}^{A B C} D^{\alpha} f\right)(t)=\left({ }^{A B C} D_{b}^{\alpha} Q f\right)(t)$ we obtain that

$$
\left({ }^{A B C} D_{b}^{\alpha} Q f\right)(t)=\left({ }^{A B R} D_{b}^{\alpha} Q f\right)(t)-\frac{B(\alpha)}{1-\alpha} f(0) E_{\alpha}\left(-\frac{\alpha}{1-\alpha}(b-t)^{\alpha}\right) .
$$

Now, we change $f(t)$ by $(Q f)(t)=f(b-t)$ to conclude our claim.

Proposition 2.9 (Integration by parts for the Caputo fractional derivative " $\left.\left(a_{a}^{A B C} D^{\alpha}\right), a=0^{\prime \prime}\right)$.

- $\left.\int_{0}^{b}\left({ }_{a}^{A B C} D^{\alpha} f\right)(t) g(t)=\int_{0}^{b} f(t)\left({ }^{A B R} D_{b}^{\alpha} g\right)(t) d t+\frac{B(\alpha)}{1-\alpha} f(t) E_{\alpha, 1, \frac{-\alpha}{1-\alpha}, b^{-}}^{1} g\right)\left.(t)\right|_{0} ^{b}$.

- $\left.\int_{0}^{b}\left(A B C D_{b}^{\alpha} f\right)(t) g(t)=\int_{0}^{b} f(t)\left({ }_{0}^{A B R} D^{\alpha} g\right)(t) d t-\frac{B(\alpha)}{1-\alpha} f(t) E_{\alpha, 1, \frac{-\alpha}{1-\alpha}, 0^{+}}^{1} g\right)\left.(t)\right|_{0} ^{b}$.

Proof. The proof of the first part follows by Theorem 2.5 and (2.6) and the proof of the second part follows by Theorem 2.5 and (2.7).

\section{The fractional Euler-Lagrange equations}

Below, we find the Euler-Lagrange equations corresponding to a Lagrangian possessing the left new Caputo derivative.

Theorem 3.1. Let $0<\alpha \leqslant 1$ be non-integer, $\mathrm{b} \in \mathbb{R}$, and $0<\mathrm{b}$. Assume that the functional $\mathrm{J}: \mathrm{C}^{2}[0, \mathrm{~b}] \rightarrow \mathbb{R}$ of the form

$$
J(f)=\int_{0}^{b} L\left(t, f(t),{ }_{0}^{A B C} D^{\alpha} f(t)\right) d t
$$

has a local extremum in $S=\left\{y \in C^{2}[0, b]: y(0)=A, y(b)=B\right\}$ at some $f \in S$, where $L:[0, b] \times \mathbb{R} \times \mathbb{R} \rightarrow \mathbb{R}$. Then,

$$
\left[\mathrm{L}_{1}(\mathrm{~s})+{ }^{\mathrm{ABR}} \mathrm{D}_{\mathrm{b}}^{\alpha} \mathrm{L}_{2}(\mathrm{~s})\right]=0, \text { for all } \mathrm{s} \in[0, \mathrm{~b}],
$$

where $\mathrm{L}_{1}(\mathrm{~s})=\frac{\partial \mathrm{L}}{\partial \mathrm{f}}(\mathrm{s})$ and $\mathrm{L}_{2}(\mathrm{~s})=\frac{\partial \mathrm{L}}{\partial_{0}^{\mathrm{ABC}} \mathrm{D}^{\alpha} \mathrm{f}}(\mathrm{s})$. 
Proof. We suppose that $J$ admits a local maximum in $S$ at $f$. Thus, there exists an $\epsilon>0$ in such a way that $J(\widehat{f})-J(f) \leqslant 0$ for all $\widehat{f} \in S$ with $\|\widehat{f}-f\|=\sup _{t \in \mathbb{N}_{a} \cap{ }_{b} \mathbb{N}}|\widehat{f}(t)-f(t)|<\epsilon$. For any $\widehat{f} \in S$ there is an $\eta \in H=\left\{y \in C^{2}[0, b], y(0)=y(b)=0\right\}$ obeying $\widehat{f}=f+\epsilon \eta$. As a result, the $\epsilon$-Taylor's theorem gives us that

$$
L(t, f, \widehat{f})=L\left(t, f+\epsilon \eta,{ }_{0}^{A B C} D^{\alpha} f+\epsilon \underset{0}{A B C} D^{\alpha} \eta\right)=L\left(t, f,{ }_{0}^{A B C} D^{\alpha} f\right)+\epsilon\left[\eta L_{1}+{ }_{0}^{A B C} D^{\alpha} \eta L_{2}\right]+O\left(\epsilon^{2}\right) .
$$

Then,

$$
\begin{aligned}
J(\widehat{f})-J(f) & =\int_{0}^{b} L\left(t, \widehat{f}(t),{ }_{0}^{A B C} D^{\alpha} \widehat{f}(t)\right) d t-\int_{0}^{b} L\left(t, f(t),{ }_{0}^{A B C} D^{\alpha} f(t)\right) d t \\
& \left.=\epsilon \int_{0}^{b}\left[\eta(t) L_{1}(t)+{ }_{0}^{A B C} D^{\alpha} \eta\right)(t) L_{2}(t)\right] d t+O\left(\epsilon^{2}\right) .
\end{aligned}
$$

Let the quantity $\delta J(\eta, y)=\int_{0}^{b}\left[\eta(t) L_{1}(t)+\left({ }_{0}^{A B C} D^{\alpha} \eta\right)(t) L_{2}(t)\right] d t$ denote the first variation of $J$.

We notice that, if $\eta \in H$, then $-\eta \in H$, and $\delta J(\eta, y)=-\delta J(-\eta, y)$. For $\epsilon$ small, the sign of $J(\widehat{f})-J(f)$ is determined by the sign of first variation, unless $\delta J(\eta, y)=0$ for all $\eta \in H$. To make the parameter $\eta$ free, we utilize the integration by part formula in Proposition 2.9, to reach

$$
\delta J(\eta, y)=\int_{0}^{b} \eta(s)\left[L_{1}(s)+{ }^{A B R} D_{b}^{\alpha} L_{2}(s)\right] d t+\left.\eta(t) \frac{B(\alpha)}{1-\alpha}\left(E_{\alpha, 1, \frac{-\alpha}{1-\alpha}, b^{-}}^{1} L_{2}\right)(t)\right|_{0} ^{b}=0,
$$

for all $\eta \in H$, and, as a result, the result is obtained by the fundamental lemma of calculus of variation.

We call $\left.\left(\mathbf{E}_{\alpha, 1, \frac{-\alpha}{1-\alpha}, b^{-}}^{1} \mathrm{~L}_{2}\right)(\mathbf{t})\right|_{0} ^{b}=0$ the natural boundary condition.

In the same manner, if the Lagrangian depends on the right Caputo fractional derivative, we obtain:

Theorem 3.2. Let $0<\alpha \leqslant 1$ be non-integer, $\mathrm{b} \in \mathbb{R}$, and $0<\mathrm{b}$. Assume that the functional $\mathrm{J}: \mathrm{C}^{2}[0, \mathrm{~b}] \rightarrow \mathbb{R}$ of the form

$$
J(f)=\int_{0}^{b} L\left(t, f(t),{ }^{A B C} D_{b}^{\alpha} f(t)\right) d t
$$

has a local extremum in $\mathrm{S}=\left\{\mathrm{y} \in \mathrm{C}^{2}[0, \mathrm{~b}]: \mathrm{y}(0)=\mathrm{A}, \mathrm{y}(\mathrm{b})=\mathrm{B}\right\}$ at some $\mathrm{f} \in \mathrm{S}$, where $\mathrm{L}:[0, \mathrm{~b}] \times \mathbb{R} \times \mathbb{R} \rightarrow \mathbb{R}$. Then,

$$
\left[\mathrm{L}_{1}(\mathrm{~s})+{ }_{0}^{\mathrm{ABR}} \mathrm{D}^{\alpha} \mathrm{L}_{2}(\mathrm{~s})\right]=0 \text { for all } \mathrm{s} \in[0, \mathrm{~b}],
$$

where $\mathrm{L}_{1}(\mathrm{~s})=\frac{\partial \mathrm{L}}{\partial \mathrm{f}}(\mathrm{s})$ and $\mathrm{L}_{2}(\mathrm{~s})=\frac{\partial \mathrm{L}}{\partial{ }^{A B C} \mathrm{D}_{\mathrm{b}}^{\alpha} \mathrm{f}}(\mathrm{s})$.

Proof. This proof is similar to Theorem 3.1 by utilizing the second integration by parts in Proposition 2.9 to obtain the natural boundary condition as $\left.\left(\mathbf{E}_{\alpha, 1, \frac{-\alpha}{1-\alpha}, 0^{+}}^{1} \mathrm{~L}_{2}\right)(\mathrm{t})\right|_{0} ^{\mathrm{b}}=0$.

Theorem 3.3 ([16]). Let $\rho, \mu, \gamma, v, \sigma, \lambda \in \mathbb{C}(\operatorname{Re}(\rho), \operatorname{Re}(\mu), \operatorname{Re}(v)>0)$, then

$$
\int_{0}^{x}(x-t)^{\mu-1} E_{\rho, \mu}^{\gamma}\left(\lambda[x-t]^{\rho}\right) t^{v-1} E_{\rho, v}^{\sigma}\left(\lambda t^{\rho}\right) d t=x^{\mu+v-1} E_{\rho, \mu+v}^{\gamma+\sigma}\left(\lambda x^{\rho}\right) .
$$

In particular, if $\gamma=1, \mu=1$, and $\rho=\alpha$, we conclude

$$
\int_{0}^{x} E_{\alpha}\left(\lambda[x-t]^{\alpha}\right) t^{v-1} E_{\alpha, v}^{\sigma}\left(\lambda t^{\alpha}\right) d t=x^{v} E_{\alpha, 1+v}^{1+\sigma}\left(\lambda x^{\alpha}\right) .
$$

From [17] we recall also the following differentiation formula that will be helpful. 
For $\alpha, \mu, \gamma, \lambda \in \mathbb{C}(\operatorname{Re}(\alpha>0)$ and $n \in \mathbb{N}$ we have

$$
\left(\frac{d}{d z}\right)^{n}\left[z^{\mu-1} E_{\alpha, \mu}^{\gamma}\left(\lambda z^{\alpha}\right)\right]=z^{\mu-n-1} E_{\alpha, \mu-n}^{\gamma}\left(\lambda z^{\alpha}\right) .
$$

Now, by the help of (3.1) and (3.2), we have

$$
{ }_{0}^{A B R} D^{\alpha}\left[\chi^{\nu-1} E_{\alpha, v}^{\sigma}\left(\lambda x^{\alpha}\right)\right]=\frac{B(\alpha)}{1-\alpha} \frac{d}{d x}\left[x^{\nu} E_{\alpha, 1+v}^{1+\sigma}\left(\lambda x^{\alpha}\right)\right]=\frac{B(\alpha)}{1-\alpha} \chi^{\nu-1} E_{\alpha, v}^{1+\sigma}\left(\lambda x^{\alpha}\right) .
$$

Similarly, by the help of (3.2) and (3.1), we have

$$
{ }_{0}^{A B C} D^{\alpha}\left[x^{v-1} E_{\alpha, v}^{\sigma}\left(\lambda x^{\alpha}\right)\right]=\frac{B(\alpha)}{1-\alpha} \int_{0}^{x} x^{v} E_{\alpha}\left(\lambda(x-t)^{\alpha}\right) \frac{d}{d t}\left[t^{v-1} E_{\alpha, v}^{\sigma}\left(\lambda x^{\alpha}\right)\right] d t=\frac{B(\alpha)}{1-\alpha} \chi^{v-1} E_{\alpha, v}^{1+\sigma}\left(\lambda x^{\alpha}\right) .
$$

Remark 3.4. An interesting observation of (3.3) and (3.4) is that the function

$$
g(x)=\lim _{\nu \rightarrow 0^{+}} \frac{1-\alpha}{B(\alpha)} x^{\nu-1} E_{\alpha, v}^{-1}\left(\lambda x^{\alpha}\right)=\frac{\alpha x^{\alpha-1}}{B(\alpha) \Gamma(\alpha)}
$$

denotes a nonzero function whose fractional $A B R$ and $A B C$ derivative become zero. This result can be seen since $(-1)_{0}=1,(-1)_{1}=-1$, and $(-1)_{k}=0$ for $k=2,3,4, \ldots$, and since

$$
\mathrm{E}_{\alpha, v}^{0}(\lambda, x)=\frac{\chi^{\nu-1}}{\Gamma(\nu)} \rightarrow 0, v \rightarrow 0^{+} .
$$

Note here that the function $\mathrm{g}(\mathrm{x})$ tends to the constant function 1 when $\alpha$ goes to 1 .

Using the following relation (14) in [9]

$$
\left({ }_{0}^{A B C} D^{\alpha} f\right)(t)=\left({ }_{0}^{A B R} D^{\alpha} f\right)(t)-\frac{B(\alpha)}{1-\alpha} f(0) E_{\alpha}\left(\lambda t^{\alpha}\right), \lambda=\frac{-\alpha}{1-\alpha},
$$

and the identity (see [17] page 78 for example)

$$
\left({ }_{0} I^{\alpha} t^{\beta-1} E_{\mu, \beta}\left[\lambda t^{\mu}\right](x)=x^{\alpha+\beta-1} E_{\mu, \alpha+\beta}\left[\lambda x^{\mu}\right],\right.
$$

where the ML-function with two parameters $\alpha$ and $\beta$ is given by

$$
\mathrm{E}_{\alpha, \beta}(z)=\sum_{k=0}^{\infty} \frac{z^{\mathrm{k}}}{\Gamma(\alpha \mathrm{k}+\beta)},(z, \beta \in \mathbb{C} ; \operatorname{Re}(\alpha)>0),
$$

where $E_{\alpha, 1}(z)=E_{\alpha}(z)$, the following result can be utilized to solve fractional dynamical systems involving the Caputo fractional derivative with ML kernels.

Proposition 3.5. For $0<\alpha<1$, we conclude that

$$
\left({ }_{a}^{A B} I^{\alpha} \underset{a}{A B C} D^{\alpha} f\right)(x)=f(x)-f(a) E_{\alpha}\left(\lambda(x-a)^{\alpha}\right)-\frac{\alpha}{1-\alpha} f(a) x^{\alpha} E_{\alpha, \alpha+1}\left(\lambda(x-a)^{\alpha}\right)=f(x)-f(a) .
$$

Similarly,

$$
\left({ }^{A B} I_{b}^{\alpha} A B C D_{b}^{\alpha} f\right)(x)=f(x)-f(b) .
$$

Example 3.6. To show the reported results we study an example of physical interest under Theorem 3.1. We deal with the following action,

$$
J(y)=\int_{0}^{b}\left[\frac{1}{2}\left(\begin{array}{l}
A B C \\
0
\end{array} D^{\alpha} y(t)\right)^{2}-V(y(t))\right] d t
$$


where $0<\alpha<1$ with $y(0), y(b)$ are assigned or with the natural boundary condition

$$
\left.\left(\mathbf{E}_{\alpha, 1, \frac{-\alpha}{1-\alpha}, b^{-}}^{1}{ }_{0}^{A B C} D^{\alpha} y(t)\right)(t)\right|_{0} ^{b}=0 .
$$

As a result, by applying Theorem 3.1 we obtain

$$
\left({ }^{A B R} D_{b}^{\alpha} \text { o }{ }_{0}^{A B C} D^{\alpha} y\right)(s)-\frac{d V}{d y}(s)=0 \text { for all } s \in[0, b] .
$$

At this stage, we conclude that it is of great interest to study the obtained Euler- Lagrange equations shown in the above example, where we have composition of right and left type fractional derivatives. For such a composition in the classical fractional case together with the action of the Q-operator we refer to [22].

Finally, we solve the above fractional Euler-Lagrange equations for certain potential functions with $\alpha=\frac{1}{2}$, and $\mathrm{B}(\alpha)=1$.

- We consider the free particle case $V \equiv 0$ : the Euler-Lagrange equations will be reduced to

$$
\left({ }^{A B R} D_{b 0}^{\alpha} A_{0}^{A B C} D^{\alpha} y\right)(t)=0 .
$$

By applying ${ }^{A B} I_{b}^{\alpha}$ to both sides we reach at

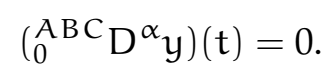

Then, by Remark 3.4 with $\mathrm{B}(\alpha)=1$ for simplicity (otherwise $\mathrm{B}(\alpha) \rightarrow 1$ as $\alpha \rightarrow 1$ ), we conclude that

$$
y(t)=c_{1}+\frac{\alpha t^{\alpha-1}}{B(\alpha) \Gamma(\alpha)}
$$

and hence using $y(0)=A$, the solution becomes

$$
y(t)=y(0)+\frac{\alpha t^{\alpha-1}}{B(\alpha) \Gamma(\alpha)}
$$

We remark here that as $\alpha \rightarrow 1$, we get the classical case.

- Let $\mathrm{V}(\mathrm{y})=c y^{2} / 2$. Then, the fractional Euler-Lagrange equations become $\left({ }^{A B R} D_{b}^{\alpha}{ }_{0}^{A B C} D^{\alpha} y\right)(t)=$ $c y(t)$. Then, applying ${ }^{A B} I_{b}^{\alpha}$ and ${ }_{0}^{A B} I^{\alpha}$, respectively together with use of (3.5), we reach at the integral equation

$$
y(t)=y(0)+c\left({ }_{0}^{A B} I^{\alpha A B} I_{b}^{\alpha} y\right)(t) .
$$

Notice that, when $\alpha$ tends to 1 we get the classical result.

\section{References}

[1] T. Abdeljawad, Dual identities in fractional difference calculus within Riemann, Adv. Difference Equ., 2013 (2013), 16 pages. 1

[2] T. Abdeljawad, On delta and nabla Caputo fractional differences and dual identities, Discrete Dyn. Nat. Soc., 2013 (2013), 12 pages. 1

[3] T. Abdeljawad, On conformable fractional calculus, J. Comput. Appl. Math., 279 (2015), 57-66. 1

[4] T. Abdeljawad, F. M. Atici, On the definitions of nabla fractional operators, Abstr. Appl. Anal., 2012 (2012), 13 pages. 1

[5] T. Abdeljawad, D. Baleanu, Fractional differences and integration by parts, J. Comput. Anal. Appl., 13 (2011), 574-582. 1

[6] T. Abdeljawad, D. Baleanu, Discrete fractional differences with nonsingular discrete Mittag-Leffler kernels, Adv. Difference Equ., 2016 (2016), 18 pages. 1 
[7] O. P. Agrawal, Formulation of Euler-Lagrange equations for fractional variational problems, J. Math. Anal. Appl., 272 (2002), 368-379. 1

[8] B. S. T. Alkahtani, Chua's circuit model with Atangana-Baleanu derivative with fractional order, Chaos Solitons Fractals, 89 (2016), 547-551. 1

[9] A. Atangana, D. Baleanu, New fractional derivative with non-local and non-singular kernel: theory and application to heat transfer model, Therm. Sci., 20 (2016), 763-769. 1, 2.1, 2, 2, 3

[10] A. Atangana, I. Koca, Chaos in a simple nonlinear system with Atangana-Baleanu derivatives with fractional order, Chaos Solitons Fractals, 89 (2016), 447-457. 1

[11] D. Baleanu, J. J. Trujillo, On exact solutions of a class of fractional Euler-Lagrange equations, Nonlinear Dynam., 52 (2008), 331-335. 1

[12] M. Caputo, M. Fabrizio, A new definition of fractional derivative without singular kernel, Progr. Fract. Differ. Appl., 1 (2015), 73-85. 1

[13] F. Gao, X.-J. Yang, Fractional Maxwell fluid with fractional derivative without singular kernel, Therm. Sci., 20 (2016), 871-877. 1

[14] R. Hilfer, Applications of fractional calculus in physics, World Sci. Publ., Singapore, (2000). 1

[15] R. Khalil, M. Al Horani, A. Yousef, M. Sababheh, A new definition of fractional derivative, J. Comput. Appl. Math., 264 (2014), 65-70. 1

[16] A. A. Kilbas, M. Saigo, K. Saxena, Generalized Mittag-Leffler function and generalized fractional calculus operators, Integral Transforms Spec. Funct., 15 (2004), 31-49. 2, 2, 3.3

[17] A. A. Kilbas, H. M. Srivastava, J. J. Trujillo, Theory and applications of fractional differential equations, North-Holland Mathematics Studies, Elsevier Science B.V., Amsterdam, (2006). 1, 2, 3, 3

[18] C. F. Lorenzo, T. T. Hartley, Variable order and distributed order fractional operators, Fractional order calculus and its applications, Nonlinear Dynam., 29 (2002), 57-98. 1

[19] J. Losada, J. J. Nieto, Properties of a new fractional derivative without singular kernel, Progr. Fract. Differ. Appl., 1 (2015), 87-92. 1

[20] J. T. Machado, V. Kiryakova, F. Mainardi, Recent history of fractional calculus, Commun. Nonlinear Sci. Numer. Simul., 16 (2011), 1140-1153. 1

[21] R. L. Magin, Fractional calculus in bioengineering, Begell House Publishers Inc, CT, (2006). 1

[22] T. Maraaba, D. Baleanu, F. Jarad, Existence and uniqueness theorem for a class of delay differential equations with left and right Caputo fractional derivatives, J. Math. Phys., 49 (2008), 11 pages. 3.6

[23] I. Podlubny, Fractional differential equations, An introduction to fractional derivatives, fractional differential equations, to methods of their solution and some of their applications, Mathematics in Science and Engineering, Academic Press, Inc., San Diego, CA, (1999). 1

[24] S. G. Samko, A. A. Kilbas, O. I. Marichev, Fractional integrals and derivatives, Theory and applications, Edited and with a foreword by S. M. Nikol'skiü, Translated from the 1987 Russian original, Revised by the authors, Gordon and Breach Science Publishers, Yverdon, (1993). 1

[25] X.-J. Yang, D. Baleanu, H. M. Srivastava, Local fractional integral transforms and their applications, Elsevier/Academic Press, Amsterdam, (2005). 1

[26] A.-M. Yang, Y. Han, J. Li, W.-X. Liu, On steady heat flow problem involving Yang-Srivastava-Machado fractional derivative without singular kernel, Therm. Sci., 20 (2016), 717-723. 1

[27] X.-J. Yang, H. M. Srivastava, J. A. Tenreiro Machado, A new fractional derivative without singular kernel: application to the modelling of the steady heat flow, Therm. Sci., 20 (2016), 753-756. 1 